\title{
MHD boundary layer flow and heat transfer over a stretching sheet with induced magnetic field.
}

\begin{abstract}
In this paper, the problem of steady magnetohydrodynamic boundary layer flow and heat transfer of a viscous and electrically conducting fluid over a stretching sheet is studied. The effect of the induced magnetic field is taken into account. The transformed ordinary differential equations are solved numerically using the finite-difference scheme known as the Keller-box method. Numerical results are obtained for various values of the magnetic parameter, the reciprocal magnetic Prandtl number and the Prandtl number. The effects of these parameters on the flow and heat transfer characteristics are determined and discussed in detail. When the magnetic field is absent, the closed analytical results for the skin friction are compared with the exact numerical results. Also the numerical results for the heat flux from the stretching surface are compared with the results reported by other authors when the magnetic field is absent. It is found that very good agreement exists.
\end{abstract}

Keyword: Boundary layer; MHD; Streching sheet. 